\title{
PBX-M ION BERNSTEIN WAVE HEATING OVERVIEW
}

M. Ono, R. Cesario ${ }^{1}$, T.K. Chu, H. Hermann, B. LeBlanc, T. Seki ${ }^{2}$, W. Tighe N. Asakura ${ }^{3}$, R. Bell, L. Blush ${ }^{4}$, S. Bernabei, R. Conn ${ }^{4}$, R. Doerner ${ }^{4}$, J. Dunlap, ${ }^{5}$ A. England $^{5}$, A. Grossman ${ }^{4}$, J. Harris ${ }^{5}$, R. Hatcher, R. Isler ${ }^{5}$, R. Kaita, S. Kaye, H. Kugel, M. Okabayashi, H. Oliver, F. Paoletti ${ }^{6}$, S. Paul, N. Sauthoff, L. Schmitz ${ }^{4}$, S. Sesnic, H. Takahashi, and G. Tynan 4 , Princeton University, Princeton, $N J,{ }^{1}$ EN JEA , Frascati, Italy, 2 NIFS Japan, ${ }^{3}$ JAERI, Japan, ${ }^{4}$ UCLA, Calif, 5 Oak Ridge National Laboratory, TN , $5_{M I T, M A}$.

\section{ABSTRACT}

A high power ion Bernstein wave heating system has been introduced on PBX-M for heating and for controlling the plasma pressure profile in an effort to achieve the stable high beta "second stability" regime. The pressure profile can be controlled through local bulk ion heating as well as density profile control. In bean-shaped plasmas with plasma currents range from $180 \mathrm{kA}$ to $250 \mathrm{kA}$, good ion heating up to the highest applied rf power, $(\approx 700 \mathrm{~kW}$, ) has been observed. The observed broadening of the ion temperature profile is consistent with localized off-axis bulk ion heating as predicted by IBW ray tracing calculations. Application of IBW also resulted in a greatly modified density profile. The ability for IBW to change the density profile appears to be particularly attractive for controlling the bootstrap current profile for advanced tokamaks. Many important IBWH-related edge physics results were also obtained, including ponderomotive edge plasma modification and parametric instability onset conditions. The experimental plan for the next IBW run includes investigation of synergy with LHCD, attainment of high bootstrap current fraction discharges utilizing the IBW density profile control, and exploration of high beta plasma regimes.

\section{INTRODUCTION}

The ion Bernstein wave (IBW) heating system (2 MW at 40-80 MHz) has been installed on PBX-M as a means of controlling the pressure profile. ${ }^{1}$ The PBX-M IBW pressure profile control strategy is centered on localized bulk ion heating 2 and density profile control. 3 The IBW system presently consists of two antennas, each connected to the $2 \mathrm{MW}, 40-80 \mathrm{MHz}$ FMIT transmitter. The IBW antenna elements are phased $(0-\pi)$ to reduce the low- $n_{\|}$related edge losses, 4 and the antennas are placed in the outer midplane regicn to optimize accessibility of IBW to the plasma core. ${ }^{5}$ The RF frequencies are 47 and $54 \mathrm{MHz}$ with $\mathrm{B}_{\mathrm{T}}=1.2 \mathrm{~T}$ and $1.4 \mathrm{~T}$, respectively, which correspond to the $5 \Omega_{\mathrm{D}}$ resonance near the plasma center.

\section{SUMMARY OF 1992 PBX-M IBW RUN RESULTS}

Heating Results - During the present run, IBW power of up to $700 \mathrm{~kW}$ was applied to plasmas with a mixture of hydrogen and deuterium. Good comparison shots with and without IBW were taken for various IBW power levels. The stored energy showed a general increase comparable to NBI at similar power levels. The temporal rise and fall of the stored energy were relatively rapid compared to the plasma density behavior. Moreover, for the experimental parameter regimes used in PBX-M, IBWH was in the saturated confinement regime, for which the stored energy is only weakly dependent on the plasma density. These observations indicate that the IBW was indeed depositing power in the core of the plasma.

To investigate the feasibility of pressure profile control by localized ion heating, it is important to check if the predicted IBW power deposition profile is consistent with the observed heating. To facilitate this comparison, the eighteen-channel charge-exchange recombination spectroscopy (CHERS) diagnostic, which uses an impurity oxygen $\left(\mathrm{O}^{+7}\right)$ line excited by a deuterium neutral beam (with a deposited NBI power of $\approx 700 \mathrm{~kW}$ ) to obtain time-resolved ion temperature profiles. The CHERS system therefore measures the bulk ion temperature, instead of the non-thermal $\mathrm{r}$ heated ion species component.

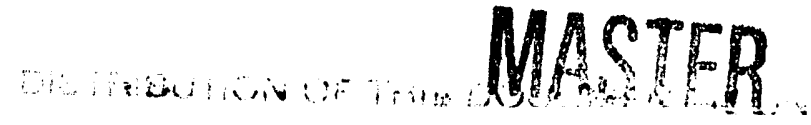


Three important ion heating results are obtained: 1$)$ the rise in the ion stored energy, ( $\left.\Delta\left[<n_{e}>T_{i}(0)\right]\right)$, is linear with the applied IBW power up to the highest powers; 2) the measured ion temperature profile shows a general broadening with a steepened gradient, $\left[d T_{i}(r) / d r\right] / T_{i}(r)$, near the predicted IBW power deposition layer as expected; and 3) a detailed examination of the earlier time shows that the ion temperature starts to rise near the power deposition layer. The TRANSP analysis of the IBW heating shows reasonable consistency with the heating result, with the usual radially-increasing $\chi_{i}$ profile. This type of localized ion heating may be utilized for a direct measurement of the local ion thermal diffusivity, $\chi_{\mathrm{i}}$. More detail is given by $\mathrm{W}$. Tighe et al. ${ }^{2}$

Particle confinement improvement and IBW-induced peaked profiles - In PBX-M, for some of the discharges, IBW has yielded very peaked density profiles. This is a clear indication of particle transport changes in the plasma core region, perhaps related to similar peaked profiles observed in JIPPT-II-U. ${ }^{6}$ The profile changes in PBX-M took place relatively slowly over $150-200$ msec. This density peaking was observed in a circular ohmic as well as in NBI-heated bean-shaped plasmas. The application of IBW into a strongly NBI-heated $\mathrm{H}$-mode discharge causes the profile to steepen from the very flat, $\mathrm{H}$-mode-like profile in the early phase to a supershot-like peaked profile in the later phase as shown in Fig. 1 . The central density reached $8 \times 10^{13} \mathrm{~cm}^{-3}$ which, without IBW, is not possible even with intense gas puffing. The density gradient reached $5 \times 10^{12} \mathrm{~cm}^{-4}$ (comparable to the $\mathrm{H}$-mode edge gradient) in the plasma core region $(\mathrm{r} \approx 10$ $15 \mathrm{~cm}$ ). This steepened density region may be related to the poloidal velocity shear stabilization of turbulence by $1 \mathrm{IBW} .{ }^{7}$ The model predicts that due a non-linear plasma response to the IBW wave field, the poloidal velocity shear layer (very much like the $\mathrm{H}$-mode case) can be created near the power absorption region. Since the wave absorption layer position can be varied, this type of study might lead to a reactor-relevant tool for active plasma transport control. With the density peaking, a complete elimination of sawteeth was observed. This suppression of sawteeth is consistent with the raising of $q(0)$ above one due to the generation of off-axis bootstrap current, as indicated by the TRANSP analysis. The details of IBW-induced transport change is discussed in the companion paper by $B$. LeBlanc at this conference. ${ }^{3}$
A CHERS diagnostic for measuring poloidal rotation during IBW is being prepared.

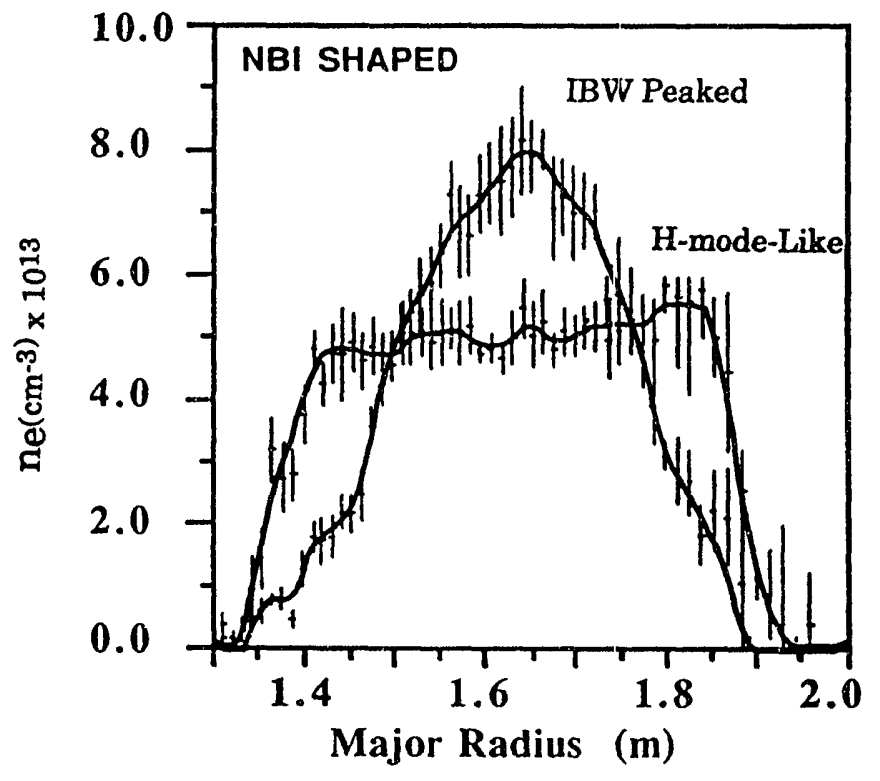

Fig. 1. Peaking of density profile during IBW. Beanshaped, $I p \approx 250 \mathrm{kA}, \mathrm{P}_{\mathrm{IBW}} \approx 500 \mathrm{~kW}$ and $\mathrm{P}_{\mathrm{NBI}} \approx 2$ $\mathrm{MW}$, deuterium, $f=47 \mathrm{MHz}$, and $B \mathrm{~T}=14.5 \mathrm{kG}$.

Edge plasma modification - To insure good heating efficiency and to reduce possible impurity generation, it is quite important to understand the edge physics occurring during IBW. This topic has been previously addre ssed by DIII-D experiments. 8 In the PBX-M IBW experiment, considerable progress has been made in understanding the IBW-edge plasma interactions, including plasma edge modification during IBW, the IBW antenna loading, and the conditions for parametric instability activity. The measured edge density during IBW by a fast reciprocating probe shows a strong reduction of the edge scrape-off density. This reduction of the density confirms the validity of the observed antenna loading based on the electron plasma wave excitation. A 
model using the ponderomotive force has shown good agreement with experimental observation. 11 The strong edge modification by IBW may be used, for example, in controlling the heat flux into the divertor plates. This reduced edge density during IBW also minimizes the parasitic antenna-plasma sheath effects during IBW.

Parametric instabilities - The topic of parametric instabilities during IBW was first addressed by the DIII-D !BW experiment. ${ }^{8}$ Associated with high power IBW, strong parametric instability activity was often observed during the DIII-D experiment, which correlated well with the edge produced high energy ion tail and electron heating. On PBX-M, this problem was investigated in detail both experimentally and theoretically. 10 Theoretical work, which includes the convective and gradient effects, shows that the parametric instability growth rate depends very strongly on the edge density profile. According to the theory, the parametric activity should increase if the plasma is moved away from the antenna of (creating a low density gap region). To test this hypothesis on PBX-M, the plasma position was deliberately varied while monitoring the parametric activity. Under the normal IBW operating conditions, very little parametric activity was observed $\leq 50 \mathrm{~dB}$ below the pump $\left(\omega \approx \omega_{\mathrm{rf}}\right)$. However, when the plasma edge was moved away from the antenna by about $10 \mathrm{~cm}$, the parametric instability activity increased to within $20 \mathrm{~dB}$ of the pump, as shown in Fig. 2.10 This result shows that the parametric instability can be controlled during IBW. The probe measurements thus far showed no sign of edge electron heating nor a significant change in the floating potential during IBW.

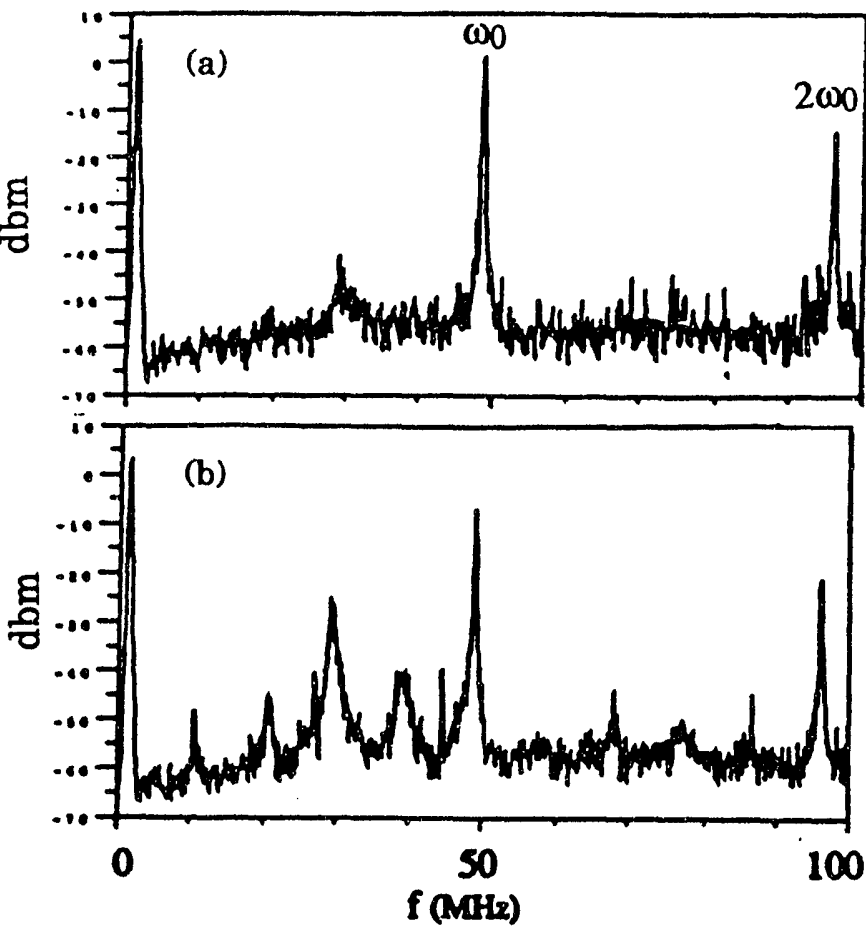

Fig. 2. Parametric instability behavior as a function of the plasma position. (a) $R=166 \mathrm{~cm}$. (b) $R=162$ cm. $\bar{n}_{e} \approx 1 \times 10^{13} \mathrm{~cm}^{-3}, P_{I B W} \approx 100 \mathrm{~kW}, I_{p}=100$ $\mathrm{kA}$, Circular, deuterium, $47 \mathrm{MHz}$ and $\mathrm{BT}_{\mathrm{T}}=14.5 \mathrm{kG}$.

\section{PLANNED IBW EXPERIMENTS ON PBX-M}

In addition to extending the present results to higher power levels with improved diagnostics, the future PBX-M IBW experimental plan has four new experimental topics: boron-nitride-clad Faraday shield, high fraction bootstrap current regime, synergy with LHCD, and ponderomotive kink stabilization.

Boron Nitride Faraday shield - In order to test the role of plasma sheaths, one of the IBW antennas was fitted with a boron-nitride-clad Faraday shield. The new shield should effectively insulate the antenna from the plasma, and thus, eliminating possible sheath related problems. ${ }^{9}$ This performance of the BN Faraday shield antenna will be compared with that of the antenna with a metallic Faraday shield.

High Fraction Bootstrap Current Regime - The density peaking during IBW produced a peak bootstrap current density contribution of $25 \%$ of the total current density ( $17 \%$ of the total current) $\left[\mathrm{T}_{\mathrm{e}}(0) \approx 1.1 \mathrm{keV}\right.$ ]. These fractions can be significantly increased with the additional available heating power (4 -6 MW NBI and 2 MW IBW). This high bootstrap current also raises $q(0)$, which is attractive for access to second 
stability regime. This bootstrap current generated in the plasma core region may be more favorable for MHD stability (particularly against kinks) compared to the H-modelike case which generates significant edge currents. The ability to produce peaked density profiles without requiring central fueling may be particularly useful in future devices such as TPX and DEMO reactors.

IBW Synergy with LHCD - The analysis of IBW on PBX-M shows that directlylaunched IBW may be utilized to enhance the quality and efficiency of the LHCD synergy. Although electron Landau damping of ion Bernstein waves is relatively weak under normal circumstances, as the wave approaches a major resonance, the electron damping increases strongly. 5 Under appropriate conditions, the localized electron Landau absorption can be quite significant. In view of the synergy with LHCD, the creation of a hot electron target at a desired location (by choosing the resonance layer position), should result in improved localization for LHCD. Moreover, since the IBW electron Landau heating essentially fills the so-called spectral gap, the LHCD efficiency is also expected to increase. If successful, this could lead to a better understanding of the JET results and an improvement of the LHCD performance in PBX-M and TPX.

IBW Ponderomotive Stabilization of External Kinks - It was recognized that the strong ponderomotive force of a low frequency slow wave launcher, such as an IBW antenna, could be used to stabilize external MHD modes such as kinks. 12 The required RF power, unfortunately, is predicted to be relatively high, making this scheme only marginally suitable for the PBX-M-type parameters. However, it was recently proposed that the use of a feedback system which senses the growth of the MHD mode (at low amplitude, $\delta B \leq 10 \mathrm{G}$ ) and modulates the antenna power in response to the mode amplitude and phase could reduce the required RF field by an order of magnitude, and the RF power by two orders of magnitude. 13 This reduction in the RF field and power requirements would make the concept reactor relevant. From a technological standpoint, the high frequency RF system is well-suited for the rapid modulation. The existing transmitters connected to the PBX-M IBW antennas are capable of RF modulation with a frequency of up to $10 \mathrm{kHz}$, permitting the test of this concept on PBX-M with only small hardware modifications.

*This work was supported by DoE Contract No. DE-AC02-76-CHO-3073.

\section{REFERENCES}

1 M. Ono, S. Bernabei, N. Asakura, et al., in the Proceedings of the Europhysics Topical Conference on Radiofrequency Heating and Current Drive of Fusion Devices, European Physical Society, 16E 213 (1992); S. Bernabei, et al., in Bull. Am. Phys. Soc. 37, 1437 (1992), to be published in Phys. Fluids B.

$2 \mathrm{~W}$. Tighe et. al., paper B 18 , this conference.

3 B. LeBlanc et al., paper B17, this conference.

$4 \mathrm{M}$. Ono, in proceedings of Ninth Topical Conference on Radio-Frequency Power in Plasmas (Charleston, 1991), AIP Conference Proceedings 244 (New York, 1992), p223.

5 M. Ono, Phys. Fluids B 5, 241 (1993).

6T. Seki, R. Kumazawa, T. Watari, et al., Nuclear Fusion, 32, 2189 (1992).

7 H. Biglari, M. Ono, P. H. Diamond, G. G. Craddock, Ref. 4, p376.

8 R.I. Pinsker, M.J. Mayberry, M. Porkolab, S. C. Chiu, and R. Prater, Ref. 4, p169.

9 A. Grossman, et al., paper B 19, this conference.

10 R. Cesario, et al., in Bull. Am. Phys. Soc. 37, 1572 (1992).

11 R. Majeski, P. Robert, T. Tanaka, et al., Bull. Am. Phys. Soc. 36, 2419 (1991).

12 D.A. D'lppolito, Phys. Fluids 31,340 (1988); J.P. Goedbloed and D.A. D'Ippolito, Phys. Fluids B2, 2366 (1990).

13 M. Okabayashi, M. Ono, D. D'Ippolito et al., the IAEA Technical Committee Meeting on the Avoidance and Control of Tokamak Disruptions, Culham, U.K., Sept. , 1991. 


\title{
PBX-M ION BERNSTEIN WAVE HEATING OVERVIEW
}

M. Ono, R. Cesario ${ }^{1}$, T.K. Chu, H. Hermann, B. LeBlanc, T. Seki ${ }^{2}$, W. Tighe N. Asakura ${ }^{3}$, R. Bell, L. Blush ${ }^{4}$, S. Bernabei, R. Conn ${ }^{4}$, R. Doerner 4 , J. Dunlap, ${ }^{5}$ A. England 5 , A. Grossman ${ }^{4}$, J. Harris ${ }^{5}$, R. Hatcher, R. Isler 5 , R. Kaita, S. Kaye, H. Kugel, M. Okabayashi, H. Oliver, F. Paoletti ${ }^{6}$, S. Paul, N. Sauthoff, L. Schmitz ${ }^{4}$, S. Sesnic, H. Takahashi, and G. Tynan ${ }^{4}$, Princeton University, Princeton, NJ, IENEA, Frascati, Italy, ${ }^{2}$ NIFS Japan, ${ }^{3}$ JAERI, Japan, ${ }^{4}$ UCLA, Calif, 5 Oak Ridge National Laboratory, TN, $5_{M I T, M A}$

\begin{abstract}
A high power ion Bernstein wave heating system has been introduced on PBX-M for heating and for controlling the plasma pressure profile in an effort to achieve the stable high beta "second stability" regime. The pressure profile can be controlled through local bulk ion heating as well as density profile control. In bean-shaped plasmas with plasma currents range from $180 \mathrm{kA}$ to $250 \mathrm{kA}$, good ion heating up to the highest applied $\mathrm{rf}$ power, $(\approx 700 \mathrm{~kW}$,) has been observed. The observed broadening of the ion temperature profile is consistent with localized off-axis bulk ion heating as predicted by IBW ray tracing calculations. Application of IBW also resulted in a greatly modified density profile. The ability for IBW to change the density profile appears to be particularly attractive for controlling the bootstrap current profile for advanced tokamaks. Many important IBWH-related edge physics results were also obtained, including ponderomotive edge plasma modification and parametric instability onset conditions. The experimental plan for the next IBW run includes investigation of synergy with LHCD, attainment of high bootstrap current fraction discharges utilizing the IBW density profile control, and exploration of high beta plasma regimes.
\end{abstract}

\section{INTRODUCTION}

The ion Bernstein wave (IBW) heating system (2 MW at 40-80 MHz) has been installed on PBX-M as a means of controlling the pressure profile. ${ }^{1}$ The PBX-M IBW pressure profile control strategy is centered on localized bulk ion heating ${ }^{2}$ and density profile control. 3 The IBW system presently consists of two antennas, each connected to the $2 \mathrm{MW}, 40-80 \mathrm{MHz}$ FMIT transmitter. The IBW antenna elements are phased $(0-\pi)$ to reduce the low- $n_{\|}$related edge losses, ${ }^{4}$ and the antennas are placed in the outer midplane region to optimize accessibility of IBW to the plasma core. 5 The RF frequencies are 47 and $54 \mathrm{MHz}$ with $\mathrm{B}_{\mathrm{T}}=1.2 \mathrm{~T}$ and $1.4 \mathrm{~T}$, respectively, which correspond to the $5 \Omega_{\mathrm{D}}$ resonance near the plasma center.

\section{SUMMARY OF 1992 PBX-M IBW RUN RESULTS}

Heating Results - During the present run, IBW power of up to $700 \mathrm{~kW}$ was applied to plasmas with a mixture of hydrogen and deuterium. Good comparison shots with and without IBW were taken for various IBW power levels. The stored energy showed a general increase comparable to NBI at similar power levels. The temporal rise and fall of the stored energy were relatively rapid compared to the plasma density behavior. Moreover, for the experimental parameter regimes used in PBX-M, IBWH was in the saturated confinement regime, for which the stored energy is only weakly dependent on the plasma density. These observations indicate that the IBW was indeed depositing power in the core of the plasma.

To investigate the feasibility of pressure profile control by localized ion heating, it is important to check if the predicted IBW power deposition profile is consistent with the observed heating. To facilitate this comparison, the eighteen-channel charge-exchange recombination spectroscopy (CHERS) diagnostic, which uses an impurity oxygen $\left(\mathrm{O}^{+7}\right)$ line excited by a deuterium neutral beam (with a deposited NBI power of $\approx 700 \mathrm{~kW}$ ) to obtain time-resolved ion temperature profiles. The CHERS system therefore measures the bulk ion temperature, instead of the non-thermal $\mathrm{rf}$ heated ion species component. 
Three important ion heating results are obtained: 1) the rise in the ion stored energy, ( $\left.\Delta\left[<n_{e}>T_{i}(0)\right]\right)$, is linear with the applied IBW power up to the highest powers; 2) the measured ion temperature profile shows a general broadening with a steepened gradient, $\left[\mathrm{dT}_{\mathbf{i}}(\mathbf{r}) / \mathrm{dr}\right] / \mathrm{T}_{\mathbf{i}}(\mathrm{r})$, near the predicted IBW power deposition layer as expected; and 3$)$ a detailed examination of the earlier time shows that the ion temperature starts to rise near the power deposition layer. The TRANSP analysis of the IBW heating shows reasonable consistency with the heating result, with the usual radially-increasing $\chi_{i}$ profile. This type of localized ion heating may be utilized for a direct measurement of the local ion thermal diffusivity, $\chi_{\mathrm{i}}$. More detail is given by $\mathrm{W}$. Tighe et al. ${ }^{2}$

Particle confinement improvement and IBW-induced peaked profiles - In PBX-M, for some 'f the discharges, IBW has yielded very peaked density profiles. This is a clear indication of particle transport changes in the plasma core region, perhaps related to similar peaked profiles observed in JIPPT-II-U. ${ }^{6}$ The profile changes in PBX-M took place relatively slowly over $150-200 \mathrm{msec}$. This density peaking was observed in a circular ohmic as well as in NBI-heated bean-shaped plasmas. The application of IBW into a strongly NBI-heated H-mode discharge causes the profile to steepen from the very flat, $\mathrm{H}$-mode-like profile in the early phase to a supershot-like peaked profile in the later phase as shown in Fig. 1. The central density reached $8 \times 10^{13} \mathrm{~cm}^{-3}$ which, without $\mathrm{IBW}_{6}$ is not possible even with intense gas puffing. The density gradient reached $5 \times 10^{12} \mathrm{~cm}^{-4}$ (comparable to the $\mathrm{H}$-mode edge gradient) in the plasma core region $(\mathrm{r} \approx 10-$ $15 \mathrm{~cm}$ ). This steepened density region may be related to the poloidal velocity shear stabilization of turbulence by IBW. 7 The model predicts that due a non-linear plasma response to the IBW wave field, the poloidal velocity shear layer (very much like the H-mode case) can be created near the power absorption region. Since the wave absorption layer position can be varied, this type of study might lead to a reactor-relevant tool for active plasma transport control. With the density peaking, a complete elimination of sawteeth was observed. This suppression of sawteeth is consistent with the raising of $q(0)$ above one due to the generation of off-axis bootstrap current, as indicated by the TRANSP analysis. The details of IBW-induced transport change is discussed in the companion paper by B. LeBlanc at this conference. 3

\section{A CHERS diagnostic for}

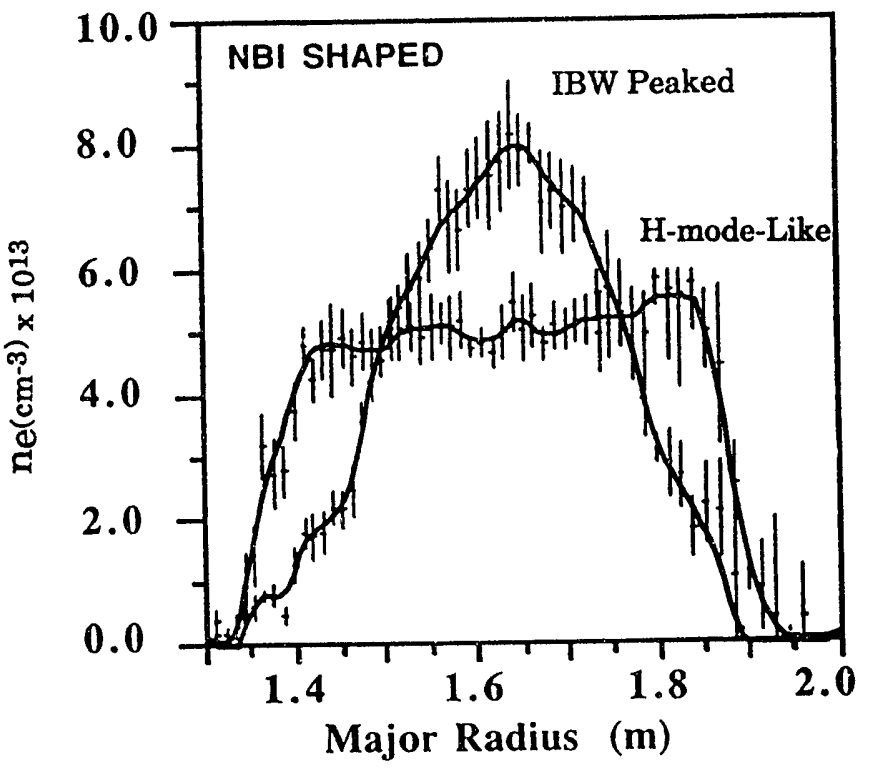

measuring poloidal rotation

Fig. 1. Peaking of density profile during IBW. Beanduring IBW is being prepared.

Edge plasma modification - To insure good heating efficiency and to reduce possible impurity generation, it is quite important to understand the edge physics occurring during IBW. This topic has been previously addressed by DIII-D experiments. ${ }^{8}$ In the PBX-M IBW experiment, considerable progress has been made in understanding the IBW-edge plasma interactions, including plasma edge modification during IBW, the IBW antenna loading, and the conditions for parametric instability activity. The measured edge density during IBW by a fast reciprocating probe shows a strong reduction of the edge scrape-off density. This reduction of the density confirms the validity of the observed antenna loading based on the electron plasma wave excitation. A 
model using the ponderomotive force has shown good agreement with experimental observation. 11 The strong edge modification by IBW may be used, for example, in controlling the heat flux into the divertor plates. This reduced edge density during IBW also minimizes the parasitic antenna-plasma sheath effects during IBW.

Parametric instabilities - The topic of parametric instabilities during IBW was first addressed by the DIII-D IBW experiment. 8 Associated with high power IBW, strong parametric instability activity was often observed during the DIII-D experiment, which correlated well with the edge-produced high energy ion tail and electron heating. On PBX-M, this problem was investigated in detail both experimentally and theoretically. 10 Theoretical work, which includes the convective and gradient effects, shows that the parametric instability growth rate depends very strongly on the edge density profile. According to the theory, the parametric activity should increase if the plasma is moved away from the antenna $\frac{0}{7}$ (creating a low density gap region). To test this hypothesis on PBX-M, the plasma position was deliberately varied while monitoring the parametric activity. Under the normal IBW operating conditions, very little parametric activity was observed $\leq 50 \mathrm{~dB}$ below the pump $\left(\omega \approx \omega_{\text {rf }}\right)$. However, when the plasma edge was moved away from the antenna by about $10 \mathrm{~cm}$, the parametric instability activity increased to within $20 \mathrm{~dB}$ of the pump, as shown in Fig. 2.10 This result shows that the parametric instability can be controlled during IBW. The probe measurements thus far showed no sign of edge electron heating nor a significant change in the floating potential during IBW.

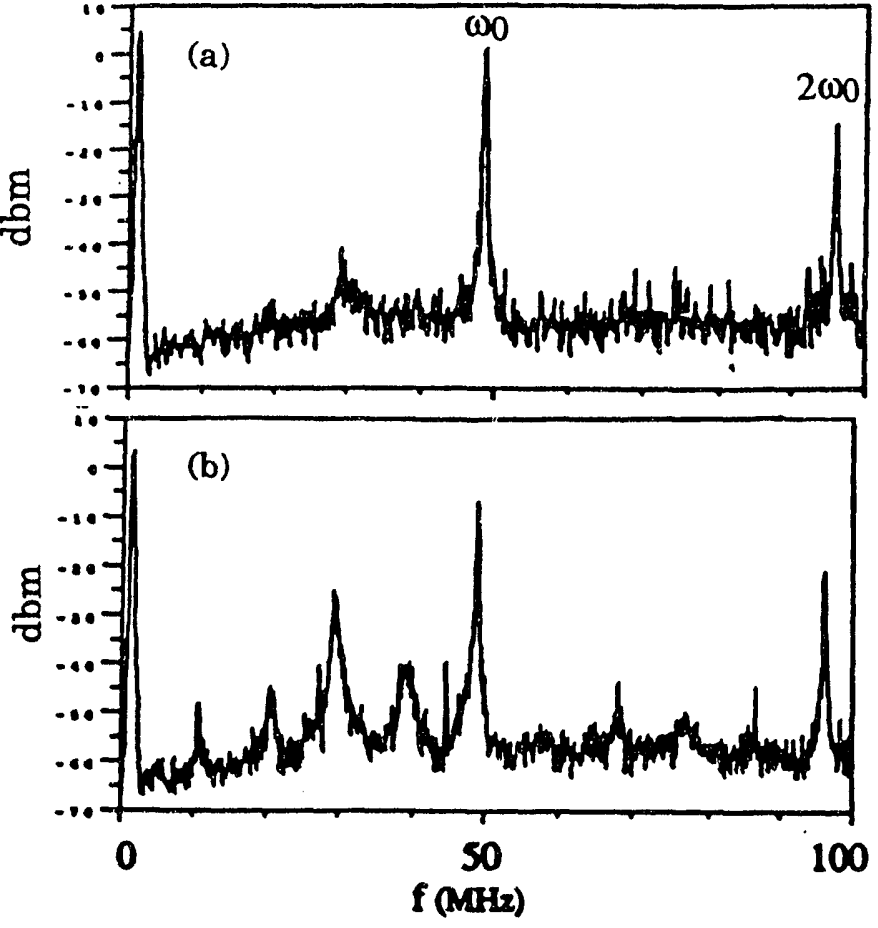

Fig. 2. Parametric instability behavior as a function of the plasma position. (a) $R=166 \mathrm{~cm}$. (b) $R=162$ $\mathrm{cm} . \overline{\mathrm{n}}_{\mathrm{e}} \approx 1 \times 10^{13} \mathrm{~cm}^{-3}, \mathrm{P}_{\mathrm{IBW}} \approx 100 \mathrm{~kW}, \mathrm{I}_{\mathrm{p}} \approx 100$ $\mathrm{kA}$, Circular, deuterium, $47 \mathrm{MHz}$ and $\mathrm{B}_{\mathrm{T}}=14.5 \mathrm{kG}$.

\section{PLANNED IBW EXPERIMENTS ON PBX-M}

In addition to extending the present results to higher power levels with improved diagnostics, the future PBX-M IBW experimental plan has four new experimental topics: boron-nitride-clad Faraday shield, high fraction bootstrap current regime, synergy with LHCD, and ponderomotive kink stabilization.

Boron Nitride Faraday shield - In order to test the role of plasma sheaths, one of the IBW antennas was fitted with a boron-nitride-clad Faraday shield. The new shield should effectively insulate the antenna from the plasma, and thus, eliminating possible sheath related problems. ${ }^{9}$ This performance of the BN Faraday shield antenna will be compared with that of the antenna with a metallic Faraday shield.

High Fraction Bootstrap Current Regime - The density peaking during IBW produced a peak bootstrap current density contribution of $25 \%$ of the total current density ( $17 \%$ of the total current) $\left[\mathrm{T}_{\mathrm{e}}(0) \approx 1.1 \mathrm{keV}\right]$. These fractions can be significantly increased with the additional available heating power (4 -6 MW NBI and 2 MW IBW). This high bootstrap current also raises $q(0)$, which is attractive for access to second 
stability regime. This bootstrap current generated in the plasma core region may be more favorable for MHD stability (particularly against kinks) compared to the $\mathrm{H}$-modelike case which generates significant edge currents. The ability to produce peaked density profiles without requiring central fueling may be particularly useful in future devices such as TPX and DEMO reactors.

IBW Synergy with L.HCD - The analysis of IBW on PBX-M shows that directlylaunched IBW may be utilized to enhance the quality and efficiency of the LHCD synergy. Although electron Landau damping of ion Bernstein waves is relatively weak under normal circumstances, as the wave approaches a major resonance, the electron damping increases strongly. 5 Under appropriate conditions, the localized electron Landau absorption can be quite significant. In view of the synergy with LHCD, the creation of a hot electron target at a desired location (by choosing the resonance layer position), should result in improved localization for LHCD. Moreover, since the IBW electron Landau heating essentially fills the so-called spectral gap, the LHCD efficiency is also expected to increase. If successful, this could lead to a better understanding of the JET results and an improvement of the LHCD performance in PBX-M and TPX.

IBW Ponderomotive Stabilization of External Kinks - It was recognized that the strong ponderomotive force of a low frequency slow wave launcher, such as an IBW antenna, could be used to stabilize external MHD modes such as kinks. 12 The required RF power, unfortunately, is predicted to be relatively high, making this scheme only marginally suitable for the PBX-M-type paraneters. However, it was recently proposed that the use of a feedback system which senses the growth of the MHD mode (at low amplitude, $\delta \mathrm{B} \leq 10 \mathrm{G}$ ) and modulates the antenna power in response to the mode amplitude and phase could reduce the required RF field by an order of magnitude, and the RF power by two orders of magnitude. 13 This reduction in the RF field and power requirements would make the concept reactor relevant. From a technological standpoint, the high frequency RF system is well-suited for the rapid modulation. The existing transmitters connected to the PBX-M IBW antennas are capable of RF modulation with a frequency of up to $10 \mathrm{kHz}$, permitting the test of this concept on PBX-M with only small hardware modifications.

*This work was supported by DoE Contract No. DE-AC02-76-CHO-3073.

\section{REFERENCES}

1 M. Ono, S. Bernabei, N. Asakura, et al., in the Proceedings of the Europhysics Topical Conference on Radiofrequency Heating and Current Drive of Fusion Devices, European Physical Society, 16E 213 (1992); S. Bernabei, et al., in Bull. Am. Phys. Soc. 37, 1437 (1992), to be published in Phys. Fluids B.

$2 \mathrm{~W}$. Tighe et. al., paper B 18 , this conference.

3 B. LeBlanc et al., paper B17, this conference.

$4 \mathrm{M}$. Ono, in proceedings of Ninth Topical Conference on Radio-Frequency Power in Plasmas (Charleston, 1991), AIP Conference Proceedings 244 (New York, 1992), p223.

5 M. Ono, Phys. Fluids B 5, 241 (1993).

6T. Seki, R. Kumazawa, T. Watari, et al., Nuclear Fusion, 32, 2189 (1992).

7 H. Biglari, M. Ono, P. H. Diamond, G. G. Craddock, Ref. 4, p376.

8 R.I. Pinsker, M.J. Mayberry, M. Porkolab, S. C. Chiu, and R. Prater, Ref. 4, p169.

9 A. Grossman, et al., paper B 19, this conference.

10 R. Cesario, et al., in Bull. Am. Phys. Soc. 37, 1572 (1992).

11 R. Majeski, P. Robert, T. Tanaka, et al., Bull. Am. Phys. Soc. 36, 2419 (1991).

12 D.A. D'lppolito, Phys. Fluids 31,340 (1988); J.P. Goedbloed and D.A. D'Ippolito, Phys. Fluids B2, 2366 (1990).

13 M. Okabayashi, M. Ono, D. D'Ir polito et al., the IAEA Technical Committee Meeting on the Avoidance and Control of Tokamak Disruptions, Culham, U.K., Sept. , 1991. 

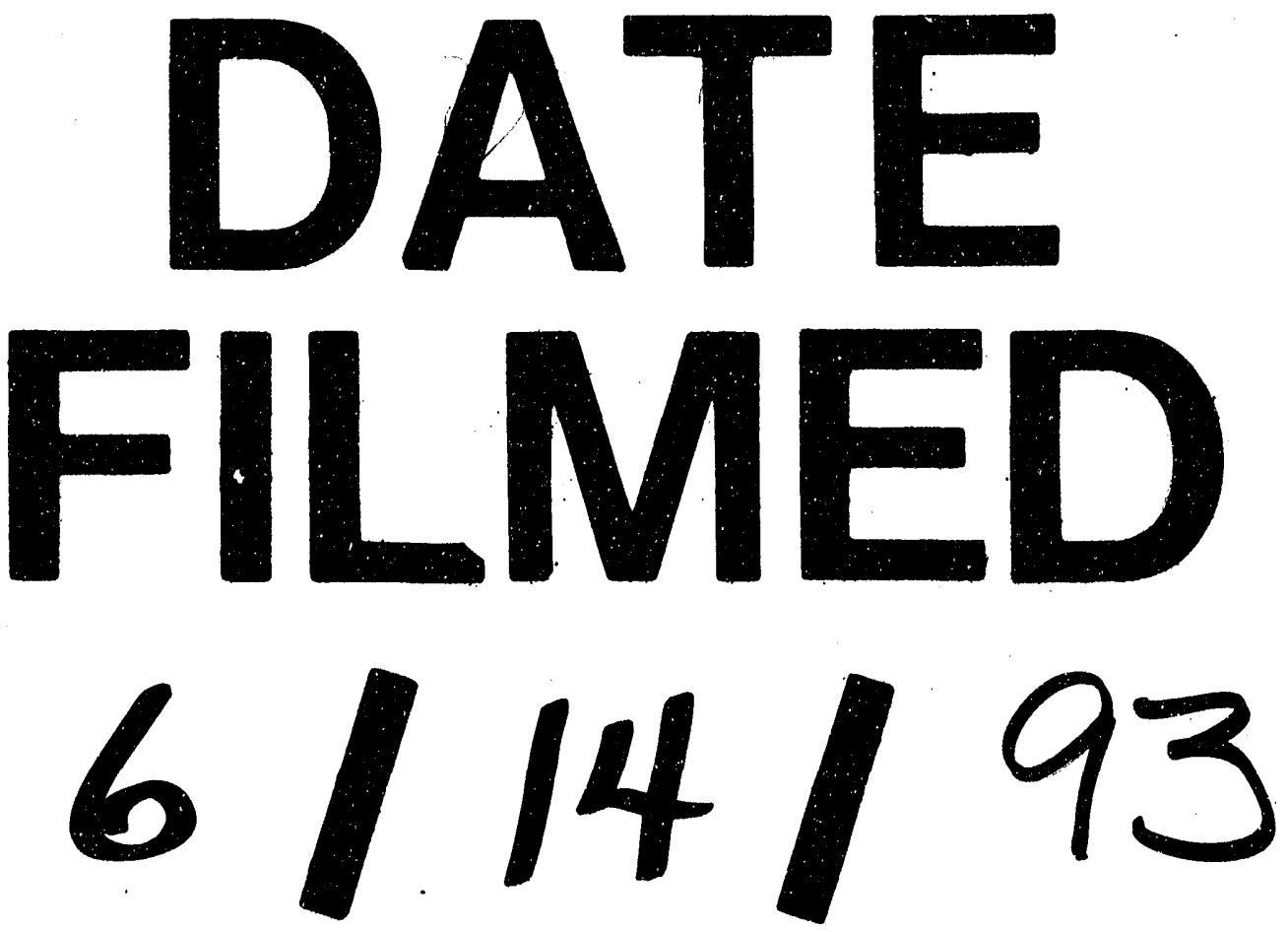
